\title{
Cognitive-behavioral therapy for body dysmorphic disorder: a review of its efficacy
}

This article was published in the following Dove Press journal:

Neuropsychiatric Disease and Treatment

27 February 2013

Number of times this article has been viewed

\author{
Angélica M Prazeres' \\ Antônio L Nascimento' \\ Leonardo F Fontenelle ${ }^{1,2}$ \\ 'Anxiety and Depression Research \\ Program, Institute of Psychiatry, \\ Universidade Federal do Rio de \\ Janeiro, Rio de Janeiro, ${ }^{2}$ Department \\ of Psychiatry and Mental Health, \\ Universidade Federal Fluminense, \\ Hospital Universitário Antonio Pedro, \\ Niterói, Brazil
}

\begin{abstract}
The aim of this study was to review the efficacy of different methods of cognitive and/or behavioral therapies used to treat body dysmorphic disorder. We evaluated all case series, open studies, controlled trials, and meta-analyses of cognitive and/or behavioral treatment approaches to body dysmorphic disorder published up to July 2012, identified through a search in the PubMed/Medline, PsycINFO, ISI Web of Knowledge, and Scopus databases. Our findings indicate that individual and group cognitive behavioral therapies are superior to waiting list for the treatment of body dysmorphic disorder. While the efficacy of cognitive therapy is supported by one controlled trial, utility of behavioral therapy is suggested by one open study and one controlled relapse prevention follow-up study. There is a pressing need to conduct head-to-head studies, with appropriate, active, control treatment groups, in order to examine further the efficacy of cognitive and/or behavioral therapies for body dysmorphic disorder.
\end{abstract}

Keywords: dysmorphophobia, behavioral therapy, cognitive therapy, literature review

\section{Introduction}

Most people are dissatisfied with some aspect of their appearance. ${ }^{1}$ In a US study, Harris and $\mathrm{Carr}^{2}$ found that, in women, the prevalence of body image concerns was highest in the age group 18-30 years (69\%) and remained high through to age 60 years $(63 \%)$. Among men, the prevalence of these concerns was highest in the age group $18-21$ years $(56 \%)$ but fell progressively with increasing age through to the age of 60 years. Even in non-Western cultures (eg, Chinese), about one third of first-year medical students $(32.5 \%)$ were reported to be very concerned about some aspect of their appearance unrelated to weight. ${ }^{3}$ Nonetheless, when a person becomes significantly distressed and/or impaired by their imaginary defects or by a small physical anomaly, a diagnosis of body dysmorphic disorder (BDD) should be considered. ${ }^{4,5}$ According to the criteria adopted by the DSM-IV-TR (Diagnostic and Statistical Manual of Mental Disorders, Fourth Edition, Text Revision), body dysmorphic disorder is classified as a somatoform disorder, although its delusional variant may also be classified as a psychotic disorder (delusional disorder, somatic type). The ICD-10 also classifies BDD as a somatoform disorder, ie, as a type of hypochondria. ${ }^{6}$

Studies have shown BDD to affect $0.7 \%-2.4 \%$ of the general population ${ }^{7,8}$ and to be associated with significant morbidity, including suicidal ideation and suicide attempts..$^{9,10}$ These figures suggest that health care providers should screen carefully for BDD, particularly because individuals with the disorder tend to be embarrassed about their perceived flaws and may be unwilling to discuss concerns about 
their appearance. ${ }^{11}$ Before undergoing treatment, a significant proportion of individuals with BDD have little or no insight, ie, they do not recognize that the flaw that they perceive is, in fact, minimal or nonexistent, or that their concerns are the result of a psychiatric disorder. ${ }^{12,13}$ In many cases, individuals with BDD have severe social and occupational dysfunction that can progress to the point of social isolation. ${ }^{14,15}$ These individuals experience shame and fear of being ridiculed, which can lead them to avoid social situations and intimate relationships. ${ }^{16} \mathrm{~A}$ diagnosis of BDD can also lead individuals to start consuming alcohol and illicit substances ${ }^{17}$ or to express safety behaviors similar to those observed in patients with social phobia. ${ }^{18,19}$

Patients with BDD frequently seek nonpsychiatric treatment, such as surgical or dermatological procedures, with the goal of eliminating the supposed flaws in their appearance. However, they are often dissatisfied with the results of esthetic procedures. ${ }^{20,21}$ These observations suggest that nonpsychiatric medical treatment generally does not improve the symptoms of BDD, and that the individual's excessive preoccupation with the apparent defect does not disappear. Recent data suggest that treatment with serotonin reuptake inhibitors $^{22}$ and cognitive and/or behavioral therapies ${ }^{23}$ is effective for BDD. Nevertheless, investigation of pharmacological and psychotherapeutic treatment of BDD is still limited, and there is a need for additional information on this topic to promote more consistent treatment guidelines.

Cognitive behavioral therapy (CBT) was built up through an integration of behavioral therapy, first popularized by Joseph Wolpe, with cognitive therapy, developed by Aaron Beck. ${ }^{24}$ While behavioral therapy emerged in the early $1950 \mathrm{~s}$ and was initially concentrated on anxiety disorders in adults, ${ }^{25}$ it was only during the 1970s that Beck's cognitive therapy gained attention as an effective treatment for patients with depression. ${ }^{26}$ Traditional behavioral therapy was not effective in depression, thus leading many behavior therapists to follow Beck's work with interest, reassured in part by the inclusion of some behavioral assignments in his cognitive program. However, it was only during the 1980s that the two streams, cognitive and behavioral, were merged into $\mathrm{CBT}{ }^{25}$

Cognitive behavioral models of BDD (reviewed by Greenberg and Wilhelm ${ }^{27}$ and Veale ${ }^{28}$ ) propose that individuals with the disorder selectively attend to minor details in their appearance, base their self worth on the way they look, react to perceived flaws with strong negative emotions, avoid social situations, and attempt to neutralize aversive feelings with ritualistic behaviors (eg, mirror checking). Most protocols target dysfunctional BDD-related thought and behavior patterns, and generally include psychoeducation, cognitive restructuring, and exposure with response prevention (ERP). Some protocols include additional modules that allow for the therapist to address BDD-specific symptoms that might not affect all patients (eg, skin picking/hair pulling, surgery-seeking, and shape/weight concerns ${ }^{29}$ ) in a flexible manner.

Several reviews of this topic have been published already. However, they have focused on studies published up to $2008,{ }^{30-32}$ on studies identified by means of an elusive methodology, ${ }^{27}$ or on studies performed in children and adolescents. ${ }^{23}$ Our paper differs from these reports by including more recent studies (up to 2012) in adults, using strict selection criteria. Although behavioral therapy, cognitive therapy, and individual and group CBT have been suggested to help patients with BDD, we believe that an updated critical review of the current level of evidence supporting their efficacy is timely.

In this study, our objective was to review the studies testing the efficacy of cognitive and/or behavioral strategies in the treatment of BDD. From this analysis, we also attempted to identify areas of research in which controlled studies have not been carried out.

\section{Materials and methods}

This review examined all studies published concerning the treatment of BDD up to July 2012. These studies were identified through a search of the PubMed/Medline, PsycINFO, ISI Web of Knowledge, and Scopus databases. Studies that involved adult patients with a primary diagnosis of BDD, according to the DSM-III, DSM-III-R, DSM-IV, or ICD-10, were included in this review. The following expressions were used in the search: "CBT" OR "cognitive behaviour therapy" OR "cognitive behavior therapy" OR "behaviour therapy" OR "behavior therapy" OR "cognitive therapy" AND ("BDD" OR "body dysmorphic disorder" OR "dysmorphophobia”). We included both terms ("behavioral" and "behavioural") in our search. The search included case series, open studies, controlled clinical trials, and meta-analytic reviews, with no restriction on publication date. We also looked at the reference sections of articles that fulfilled the selection criteria of our studies.

Narrative reviews, editorials, letters, single case studies, and treatment guidelines were excluded. Only studies published in English, French, Spanish, and Portuguese were evaluated. These limits were determined using the existing filters in the PubMed/Medline and PsycINFO databases and by subsequent inspection of articles by a psychologist (AP). 


\section{Results}

Our initial criteria identified 113 articles in the ISI Web of Knowledge, 140 articles in PubMed/Medline, 165 articles in Scopus, and 16 articles in PsycINFO. After analyzing each of the four databases according to the inclusion criteria and excluding duplicates of articles that had been found in more than one database, we selected a total of 14 articles. Articles that were not included in this review either did not include patients with a primary diagnosis of BDD, did not focus on psychological interventions, did not concentrate on cognitive or behavioral therapy, or were published in Japanese or German. The studies found by our search are listed in Tables 1-3. A more detailed description of each type of study is provided in the following sections.

\section{Individual CBT}

An open study ${ }^{33}$ was performed to assess the efficacy of individual CBT in patients with BDD. Seventeen patients diagnosed with DSM-III-R BDD (75\% of whom had at least four different types of personality disorder) received 4 weeks of individual intensive $\mathrm{CBT}$, which was carried out five times a week in 90 -minute sessions. Sessions were divided into 60 minutes of ERP and 30 minutes of cognitive therapy. At the end of 4 weeks of intensive treatment, the patients showed statistically significant reductions in their initial scores on the Modified Yale Brown Obsessive Compulsive Scale for BDD (YBOCS-BDD).

In another study, ${ }^{34}$ the authors randomly assigned 19 patients diagnosed with BDD (according to DSM-IV criteria) to individual CBT or to a control waiting list for a period of 12 weeks. The CBT proposed in this study focused on cognitive restructuring (related to perception of body image, beliefs or attitudes related to body image, and attention to self-references) as well as ERP. The control group (10 patients) did not present statistically significant differences on any of the measures evaluated before and after 12 weeks. In contrast, the group of patients that received CBT showed statistically significant improvements on measures of depression (Hospital Depression Score and the Montgomery Asberg Depression Rating Scale) and BDD (YBOCS-BDD and Body Dysmorphic Disorder Examination).

More recently, Wilhelm et $\mathrm{al}^{29}$ undertook an open study to test a broadly applicable "modular" form of CBT developed specifically to address the unique symptoms of BDD and its associated attributes (involving, eg, skin picking and hair pulling, muscularity and shape, cosmetic treatment, and mood) in a flexible yet structured way. Treatment was delivered in weekly individual sessions over 18 or 22 weeks.
Standardized clinician ratings and self-report measures were used to assess BDD (YBOCS-BDD) and related symptoms (Clinical Global Impression, Brown Assessment of Beliefs Scale, Beck Depression Inventory, and Client Satisfaction Inventory) before and after treatment and at 3-month and 6-month follow-up appointments. Following treatment, BDD and associated depressive symptoms showed statistically significant decreases in severity, with $80 \%$ of completers considered to be responders (decrease on the YBOCS-BDD initial scores greater than 30\%). Treatment gains were maintained at follow-up. Low dropout rates (20\%) and very high patient satisfaction ratings (88.9\%) suggest that the treatment was highly acceptable to patients.

In sum, we were able to find three studies on individual $\mathrm{CBT}$ in BDD, ie, two open and one using a waiting list as a control. All supported the efficacy of individual CBT in BDD. Preliminary evidence suggests that CBT is useful in samples with comorbid personality disorder and as a "modular" strategy to treat the different problems associated with the disorder.

\section{Group CBT}

Wilhelm et $\mathrm{al}^{35}$ analyzed a case series that included 13 patients with BDD who were treated with group CBT in 12 weekly sessions of 90 minutes each. The protocol involved psychoeducation, identification of thoughts and behaviors related to $\mathrm{BDD}$, cognitive restructuring, ERP, and insertion into pleasurable situations, as directed by the therapist. Two patients discontinued treatment after the first session and their data were excluded from the sample. Statistically significant improvements, including reduction in both BDD symptoms (according to the YBOCS-BDD) and severity of depression (according to the Beck Depression Inventory) were observed among those individuals who completed treatment. However, because there was no control group, it was not possible to attribute the improvements to group CBT alone.

The first controlled study to evaluate the efficacy of group CBT in the treatment of BDD was conducted by Rosen et al, ${ }^{36}$ who assessed a total of 54 individuals. These participants were randomized to receive group CBT or to a waiting list. Twenty-seven patients were treated in small groups in eight 2-hour sessions. Group CBT included modification of intrusive thoughts related to body dissatisfaction and overvaluation of physical appearance, exposure to avoided situations related to body image, and elimination of body checking compulsions (ie, ERP). Individuals in the treatment group showed statistically significant decreases in severity of BDD symptoms, as compared with patients on 


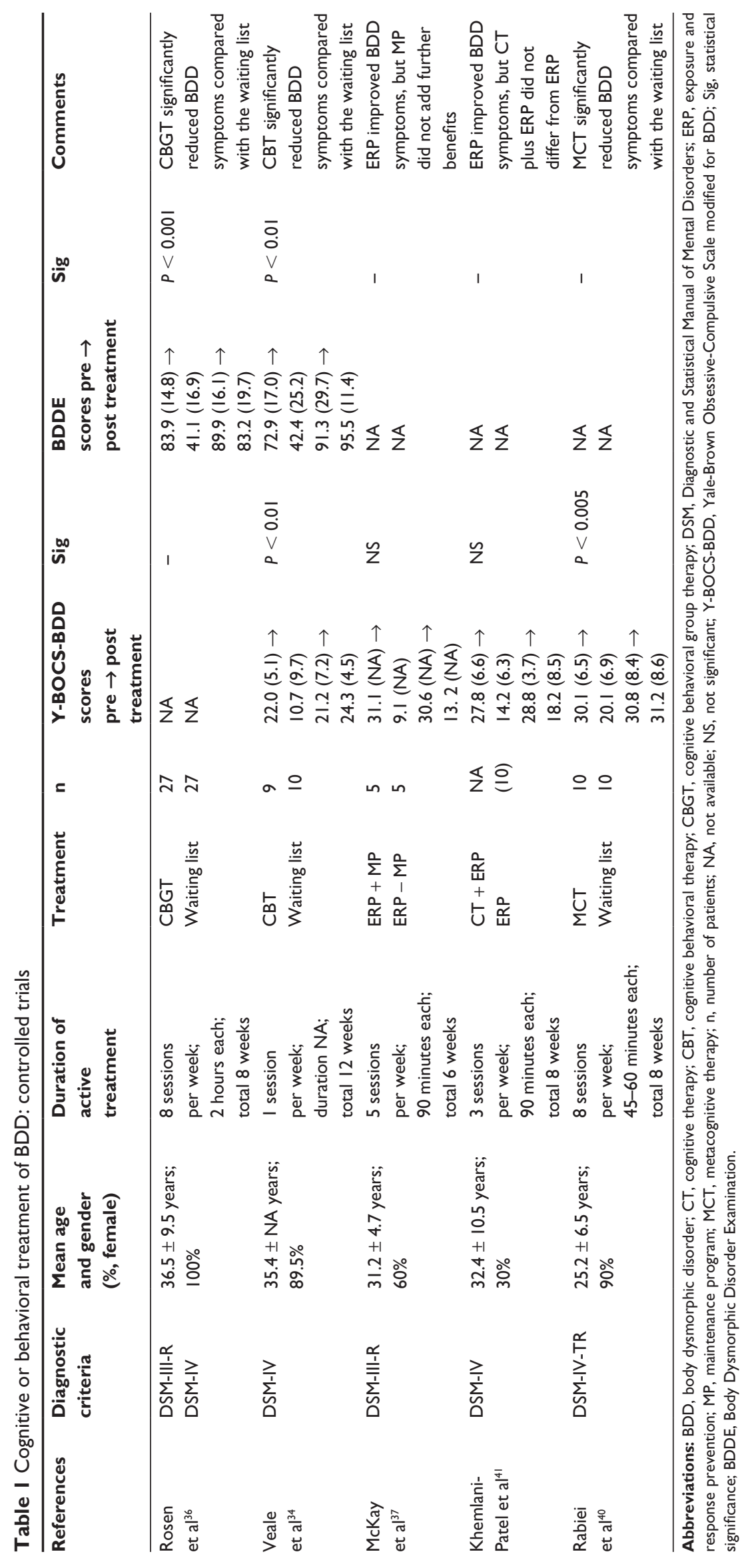


the waiting list, who did not receive any type of treatment. Post-treatment measures revealed that symptoms of BDD (according to the Body Dysmorphic Disorder Examination) decreased significantly in $82 \%$ of cases; follow-up showed the same results for $77 \%$ of participants. In sum, we found two studies on group CBT in BDD, ie, one open and one using a waiting list as a control. Both studies supported the efficacy of group CBT in BDD.

\section{Behavioral therapy}

A single open study ${ }^{37}$ evaluated the effects of behavioral therapy in 10 patients diagnosed with BDD using ERP. Behavioral therapy was administered during 6 consecutive months in five 90-minute sessions per week. Both live and imaginary ERP sessions were used. The results in general indicated that patients responded well to ERP, and they showed statistically significant reductions in scores of assessment instruments related to BDD (YBOCS-BDD), avoidance (Behavioral Avoidance Test), depression (Beck Depression Inventory), and anxiety symptoms (Beck Anxiety Inventory).

In the same study protocol, but after acute treatment, patients were randomly allocated to either a maintenance program $(n=5)$ or to a group that did not receive treatment $(n=5)$. The maintenance program consisted of a standard psychoeducation session focusing on topics such as relapse, lapses or fluctuations in symptoms, strategies for managing symptoms, and maintenance ERP. Patients were re-evaluated after 6 months. Patients who participated in the maintenance program had significantly lower levels of depression and anxiety at endpoint, were able to keep the gains they had made, and maintained statistically significant reductions in their levels of depression, anxiety, and BDD symptoms during the following 6 months.

During the subsequent 2 years, the same authors performed a follow-up analysis ${ }^{38}$ of the differences between the maintenance program group and the control group. These groups were evaluated at 12,18 , and 24 months following the end of the maintenance program, or, in the case of the control group, 6 months after the end of the treatment phase. At the end of 2 years, the patients who participated in the maintenance program were better able to manage fluctuations in their symptoms and to prevent relapses. In addition, they showed significantly fewer symptoms of anxiety and depression when compared with the control group. However, both groups maintained improvement in acute BDD symptomatology and behavioral avoidance obtained during the treatment phase.
In sum, we found one single study on behavioral therapy in BDD that included an initial open treatment phase followed by a controlled segment involving maintenance treatment versus no maintenance treatment. While the first open part suggested the usefulness of behavioral therapy in the short term, the controlled part confirmed the efficacy of behavioral therapy for preventing relapses on a long-term basis.

\section{Cognitive therapy}

A single open study ${ }^{39}$ evaluated the effect of cognitive therapy on symptoms in four patients diagnosed with BDD. In this study, cognitive therapy differed from CBT by not providing any behavioral instructions to patients with BDD. Patients underwent 3-5 weeks of baseline data collection, 7 weeks of individual cognitive therapy, and 3-5 weeks of follow-up data collection (total of 15 weeks). During the initial phases of data collection and follow-up, patients had sessions once per week. During the cognitive therapy phase, participants attended two sessions per week, each of which lasted for 75 minutes. At the end of treatment, three of the four patients (patients 1, 2, and 3) showed a statistically significant reduction in depression and anxiety, as shown by Beck Depression and Anxiety Inventory scores and in dissatisfaction with parts of their bodies (patients 1, 2, and 4) according to the Body Satisfaction Scale. There were also statistically significant decreases in BDD-related obsessions [on patients $1(14 \rightarrow 6)$, $2(11 \rightarrow 5)$, and $3(17 \rightarrow 3)$ ] and compulsions [on patients $1(14 \rightarrow 4)$ and $3(14 \rightarrow 3)$ ] according to the YBOCS-BDD scores. Two patients also showed a significant reduction in overvalued ideas (patients 1 and 4) as shown by scores on the Overvalued Ideas Scale.

In a controlled study, Rabiei et $\mathrm{al}^{40}$ investigated the efficacy of metacognitive intervention in 20 patients with BDD. Subjects were randomly assigned to 8 weeks of either metacognitive intervention or to a waiting list control group. The aim of metacognitive intervention was to enable patients to treat their thoughts simply as mental events by modifying metacognitive beliefs about appearance and rituals, reducing levels of self-focused attention and rumination, and developing adaptive coping strategies. The Y-BOCS-BDD and the Thought-Fusion Inventory were administered at pretest, posttest (after 2 months), and follow-up (after 6 months). The results demonstrated that metacognitive intervention led to statistically significant reductions in symptoms of BDD and of thoughtfusion compared with the waiting list group. Effects on both outcome measures were maintained at 6-month follow-up.

In sum, we were able to find two studies of cognitive therapy in $\mathrm{BDD}$, one open and the other using waiting list 
Table 2 Cognitive or behavioral treatment for BDD: case series and open trials

\begin{tabular}{|c|c|c|c|c|c|c|c|c|c|}
\hline References & $\begin{array}{l}\text { Type of } \\
\text { study }\end{array}$ & $\begin{array}{l}\text { Diagnostic } \\
\text { criteria }\end{array}$ & $\begin{array}{l}\text { Age and } \\
\text { gender } \\
\text { (\% female) }\end{array}$ & $\begin{array}{l}\text { Duration of } \\
\text { treatment }\end{array}$ & Treatment & $\mathbf{n}$ & $\begin{array}{l}\text { Y-BOCS- } \\
\text { BDD scores } \\
\text { pre } \rightarrow \text { post } \\
\text { treatment }\end{array}$ & Sig & $\begin{array}{l}\text { General } \\
\text { comments }\end{array}$ \\
\hline $\begin{array}{l}\text { Neziroglu } \\
\text { and Yaryura- } \\
\text { Tobias }^{46}\end{array}$ & $\begin{array}{l}\text { Case } \\
\text { series }\end{array}$ & DSM-III-R & $\begin{array}{l}24.6 \pm N A \\
\text { years; } 60 \%\end{array}$ & $\begin{array}{l}\text { I-5 sessions } \\
\text { per week, } \\
90 \text { minutes } \\
\text { each } \\
\text { (total I2-48 } \\
\text { sessions) }\end{array}$ & $\begin{array}{l}\text { Intensive CBT or } \\
\text { weekly CBT }\end{array}$ & 5 & NA & NA & $\begin{array}{l}\text { Authors note } \\
\text { improvement } \\
\text { of BDD } \\
\text { symptoms } \\
\text { (including } \\
\text { overvalued } \\
\text { ideas) in four } \\
\text { patients }\end{array}$ \\
\hline $\begin{array}{l}\text { Wilhelm } \\
\text { et } \mathrm{al}^{35}\end{array}$ & $\begin{array}{l}\text { Case } \\
\text { series }\end{array}$ & DSM-IV & $\begin{array}{l}30.6 \pm 10.9 \\
\text { years; } 76.9 \%\end{array}$ & $\begin{array}{l}\text { I session } \\
\text { per week, } \\
90 \text { minutes } \\
\text { each } \\
\text { (total } 12 \\
\text { sessions) }\end{array}$ & CBGT & 13 & $\begin{array}{l}28.5(\mathrm{NA}) \rightarrow \\
21.3(\mathrm{NA})\end{array}$ & $\begin{array}{l}t(7)=3.6 \\
P<0.01\end{array}$ & $\begin{array}{l}\text { High levels } \\
\text { of attrition } \\
(31 \%) \text { were } \\
\text { reported } \\
\text { this sample }\end{array}$ \\
\hline $\begin{array}{l}\text { Saxena } \\
\text { et al }{ }^{42}\end{array}$ & $\begin{array}{l}\text { Case } \\
\text { series }\end{array}$ & DSM-III-R & $\begin{array}{l}32.5 \pm 8.5 \\
\text { years; } 54.4 \%\end{array}$ & $\begin{array}{l}6 \text { weeks } \\
\text { (other } \\
\text { information } \\
\text { NA) }\end{array}$ & $\begin{array}{l}\text { Intensive CBT, } \\
\text { medication, and } \\
\text { psychosocial } \\
\text { rehabilitation }\end{array}$ & II & $\begin{array}{l}29.6(3.7) \rightarrow \\
16.9(5.1)\end{array}$ & NA & $\begin{array}{l}\text { BDD showed } \\
\text { similar patterns } \\
\text { of response } \\
\text { to OCD. } \\
\text { Authors note } \\
\text { greater decreases } \\
\text { in anxiety and } \\
\text { depression in } \\
\text { BDD than } O C D\end{array}$ \\
\hline $\begin{array}{l}\text { Neziroglu } \\
\text { et } \mathrm{al}^{33}\end{array}$ & $\begin{array}{l}\text { Open } \\
\text { trial }\end{array}$ & DSM-III-R & $\begin{array}{l}25.1 \pm 10.3 \\
\text { years; } 58.8 \%\end{array}$ & $\begin{array}{l}5 \text { sessions } \\
\text { per week, } \\
90 \text { minutes } \\
\text { each } \\
\text { (total } 20 \\
\text { sessions) }\end{array}$ & Intensive CBT & 17 & $\begin{array}{l}25.7(\mathrm{NA}) \rightarrow \\
12.0(\mathrm{NA})\end{array}$ & $\begin{array}{l}t(16)=6.4 \\
P<0.001\end{array}$ & $\begin{array}{l}\text { OCD was } \\
\text { present in } 94 \% \\
\text { of this sample. } \\
\text { Over } 75 \% \text { of } \\
\text { the patients had } \\
\text { more than four } \\
\text { Axis II diagnoses }\end{array}$ \\
\hline $\begin{array}{l}\text { McKay } \\
\text { et a }\left.\right|^{37}\end{array}$ & $\begin{array}{l}\text { Open } \\
\text { trial }\end{array}$ & DSM-III-R & $\begin{array}{l}31.2 \pm 4.7 \\
\text { years; } 60 \%\end{array}$ & $\begin{array}{l}5 \text { sessions } \\
\text { per week } \\
90 \text { minutes } \\
\text { each } \\
\text { (total NA) }\end{array}$ & ERP & 10 & $\begin{array}{l}31.1(\mathrm{NA}) \rightarrow \\
9.1 \text { (NA) }\end{array}$ & $\begin{array}{l}t(9)=5.5 \\
P<0.001\end{array}$ & $\begin{array}{l}\text { Exposure was } \\
\text { either in vivo or } \\
\text { in imagery }\end{array}$ \\
\hline $\begin{array}{l}\text { Geremia } \\
\text { and } \\
\text { Neziroglu }{ }^{39}\end{array}$ & $\begin{array}{l}\text { Open } \\
\text { trial }\end{array}$ & DSM-IV & $\begin{array}{l}31.2 \pm 4.5 \\
\text { years; } 50 \%\end{array}$ & $\begin{array}{l}2 \text { sessions } \\
\text { per week, } \\
75 \text { minutes } \\
\text { each } \\
\text { (total } 30 \\
\text { sessions) }\end{array}$ & CT & 4 & $\begin{array}{l}25.2(4.3) \rightarrow \\
11.0(3.9)\end{array}$ & NA & $\begin{array}{l}\text { High levels of } \\
\text { compliance to } \\
\text { assignments } \\
(100 \%) \text { contrasts } \\
\text { with the low } \\
\text { compliance rates } \\
\text { in the strictly } \\
\text { behavioral } \\
\text { treatment } \\
\text { protocol }\end{array}$ \\
\hline $\begin{array}{l}\text { Wilhelm } \\
\text { et } \mathrm{al}^{29}\end{array}$ & $\begin{array}{l}\text { Open } \\
\text { trial }\end{array}$ & DSM-IV & $\begin{array}{l}32.2 \pm 4.6 \\
\text { years; } 58 \%\end{array}$ & $\begin{array}{l}\text { I-2 sessions } \\
\text { per week, } \\
60 \text { minutes } \\
\text { each } \\
\text { (total I8-22 } \\
\text { sessions) }\end{array}$ & Modular CBT & 12 & $\begin{array}{l}32.9(4.1) \rightarrow \\
18.1(11.7)\end{array}$ & $\begin{array}{l}t(I I)=5.4 \\
P<0.00 I\end{array}$ & $\begin{array}{l}\text { More than } 80 \% \text { of } \\
\text { those who began } \\
\text { modular CBT } \\
\text { completed it }\end{array}$ \\
\hline
\end{tabular}

Abbreviations: BDD, body dysmorphic disorder; NA, not available; CBT, cognitive behavioral therapy; CBGT, group cognitive behavioral therapy; CT, cognitive therapy; DSM, Diagnostic and Statistical Manual of Mental Disorders; n, number of patients; OCD, obsessive-compulsive disorder; OCS, obsessive-compulsive symptoms. 
Table 3 Cognitive or behavioral treatment for BDD: meta-analysis

\begin{tabular}{|c|c|c|c|}
\hline References & Treatment assessed & Studies analyzed (n) & Response \\
\hline Williams et $\mathrm{a}^{31}$ & $\begin{array}{l}\text { Pharmacotherapy (clomipramine, } \\
\text { fluvoxamine, fluoxetine, citalopram) } \\
\text { and CBT (ERP; CT; ERP + CT) }\end{array}$ & $\begin{array}{l}\text { I5 studies } \\
\text { I. Five pharmacological } \\
\text { (I RCT; } 3 \text { case series; I crossover) } \\
\text { 2. I0 CBT ( } 2 \text { RCT; } 8 \text { case series) }\end{array}$ & $\begin{array}{l}\text { Both approaches were effective with } \\
\text { small advantage of CBT in reduction } \\
\text { of severity of BDD symptoms }\end{array}$ \\
\hline Ipser et al ${ }^{32}$ & $\begin{array}{l}\text { Pharmacotherapy (clomipramine } \\
\text { versus desipramine and fluoxetine } \\
\text { versus placebo) and CBT (ERP; } \\
\text { ERP }+C T \text { ) }\end{array}$ & $\begin{array}{l}5 \text { studies } \\
\text { I. Two pharmacological ( } 2 \text { RCT) } \\
\text { 2. Three CBT ( } 3 \text { RCT) }\end{array}$ & $\begin{array}{l}\text { Both approaches were effective, with } \\
\text { no advantage of CBT. Differences in } \\
\text { study design prohibited synthesis of } \\
\text { data from pharmacotherapy trials }\end{array}$ \\
\hline
\end{tabular}

Abbreviations: $n$, number of samples; ERP, exposure and response prevention; CT, cognitive therapy; RCT, randomized controlled trial; $C B T$, cognitive behavioral therapy; BDD, body dysmorphic disorder.

as a control. While the open study suggested the efficacy of cognitive therapy in BDD, the controlled one confirmed the efficacy of a specific type of cognitive therapy, namely metacognitive intervention, in the disorder.

\section{Behavioral therapy versus CBT}

Khemlani-Patel et $\mathrm{al}^{41}$ compared 10 patients randomly assigned to ERP or CBT in order to evaluate whether addition of cognitive strategies improves the treatment response in BDD patients. Both intervention conditions were conducted three times a week for a total of 24 sessions in 8 weeks. The CBT protocol consisted of 4 weeks of cognitive treatment followed by 4 weeks of in vivo ERP. Patients with BDD assigned to ERP treatment received 8 weeks of in vivo ERP alone. After treatment, the results indicated that ERP was equally effective as CBT, and groups displayed statistically significant decreases on measures of BDD (YBOCS-BDD and Body Dysmorphic Disorder Examination), anxiety (Beck Anxiety Inventory), and depression (Beck Depression Inventory II) after 2 weeks of baseline and 4 weeks of intensive treatment, regardless of treatment condition. In addition, neither group improved on satisfaction with body parts (based on the Defect Related Beliefs Test), indicating that although the participants improved on functioning, degree of distress, and time spent on BDD, they did not necessarily view their appearance as being significantly different. The authors suggest that cognitive therapy is at least as effective as behavior therapy but does not necessarily enhance treatment outcome for BDD.

\section{CBT, medication, and psychosocial rehabilitation during partial hospitalization}

While the treatment guidelines for BDD are similar to those for obsessive-compulsive disorder, ${ }^{42}$ patient responses to treatment have not been directly compared until recently.
A retrospective study ${ }^{42}$ assessed 107 patients with DSMIII-R obsessive-compulsive disorder $(\mathrm{n}=96)$ or BDD $(n=11)$ who were treated openly for 6 weeks with intensive CBT, medication, and psychosocial rehabilitation in a partial hospitalization program specializing in treatment for patients with severe obsessive-compulsive disorder. All patients were evaluated before and after their treatments for obsessivecompulsive (Y-BOCS), depression (Hamilton Depression Rating Scale), anxiety (Hamilton Anxiety Rating Scale), and global (Global Assessment Scale) symptoms. Patients with BDD showed more severe symptoms of anxiety and depression during the initial evaluation. The proportion of patients treated with selective serotonin reuptake inhibitors and antipsychotic medications did not differ between the groups. Both groups showed statistically significant improvements in obsessive and/or compulsive symptoms, anxiety, and depression. However, the amount of improvement in measures of anxiety and depression was significantly greater in patients with BDD than in patients with obsessivecompulsive disorder.

\section{Meta-analysis}

Two meta-analyses were conducted to investigate the efficacy of pharmacotherapy and CBT as a treatment for BDD, and both yielded similar results, suggesting that both medication and CBT may effectively reduce symptoms of BDD. The first meta-analysis ${ }^{31}$ evaluated 15 studies, including case series and randomized clinical trials published between 1994 and 2003; six of these studies involved pharmacological treatment (clomipramine, desipramine, fluvoxamine, fluoxetine, and citalopram) and nine involved psychotherapy (cognitive therapy, ERP, and CBT). The results confirmed the efficacy of both treatment methods in improving the symptoms of BDD and depression, suggesting an advantage of CBT over pharmacotherapy in reduction of the severity of BDD symptoms. 
Similar results were obtained in a more recent metaanalysis, ${ }^{32}$ which included five randomized trials that analyzed data from 169 subjects from two studies on pharmacotherapy (clomipramine versus desipramine, fluoxetine versus placebo) and three studies on CBT (CBT versus waiting list; $\mathrm{CBT}$ versus control group; $\mathrm{CBT}$ versus $\mathrm{CBT}+\mathrm{SP}$ ). Both selective serotonin reuptake inhibitors and $\mathrm{CBT}$ proved to be effective treatments for patients with BDD after taking the response, adherence, and dropout rates into consideration. In addition, they found preliminary evidence from one trial suggesting that the gains obtained in CBT were maintained after the end of treatment (relapse rate 4/22). Nevertheless, the authors were unable to confirm any superiority of CBT over pharmacotherapy, and suggested that differences in study design prohibited synthesis of data from pharmacotherapy trials.

\section{Discussion}

The studies described in this review, although methodologically heterogeneous, suggest that individual and group CBT are empirically supported treatments for BDD. However, most controlled studies were restricted to comparisons with groups that were not submitted to an active treatment (eg, waiting list). Therefore, while CBT strategies may be the most studied forms of psychotherapy for BDD, ${ }^{43}$ it is still not completely clear whether their therapeutic effects are secondary to specific CBT techniques or to nonspecific effects of the doctor-patient relationship.

Two open studies and at least one controlled study (using a waiting list) have suggested that individual CBT is an effective treatment for BDD. The combination of ERP and cognitive therapy (ie, CBT) has been shown to be potentially effective in BDD in different forms, ie, weekly or intensive CBT, CBT associated with role playing, "modular" CBT, and CBT associated with medication or psychosocial rehabilitation, even among patients who present comorbidity with various personality disorders. Nevertheless, one controlled study suggests that cognitive therapy added to ERP does not result in significant gains as compared with pure ERP. Patients treated with individual CBT showed decreased concern with their imagined physical defects, their overvalued and delusional ideas, their obsessive and compulsive symptoms, anxiety, and depression, and their performance in professional and social activities.

One open study and one controlled study (using a waiting list) revealed favorable effects of group CBT in the treatment of BDD, both in relation to symptomatology of the disorder and in relation to comorbid depression. These results reinforce the importance of controlled studies that investigate the use of group CBT for the treatment of BDD because this method of treatment may be financially advantageous for the public health system, in that it allows for treatment of a greater number of patients at a lower cost. However, it might also chase some BDD patients with prominent social anxiety.

Evidence of the efficacy of purely behavioral or cognitive techniques is still insufficient because the evidence is based primarily on open studies. For example, behavioral therapy involving imaginary exposure or live exposure proved to be effective in the reduction of BDD symptoms, avoidance behavior, depression, and anxiety in just one open study followed by a controlled relapse prevention phase. The decrease in symptoms achieved with ERP was more easily maintained when accompanied by psychotherapy that focused on maintaining post-treatment gains. This suggests that the maintenance phase is an important component of treatment that prevents relapses and helps the patient self-manage fluctuations in symptoms typically associated with BDD.

Similarly, cognitive therapy effectively reduced patient levels of depression and anxiety, obsessive-compulsive symptoms related to BDD, delusional beliefs, and overvaluation of body parts in one open study and in one controlled study (using a metacognitive intervention). In fact, even though cognitive therapy has a specifically cognitive focus, it may spontaneously generate behavioral effects for some patients, who begin to expose themselves to various social situations (eg, school, work, and daylight) as a consequence of feeling less dissatisfied with their "defect". Exposure to social situations may have had a dual effect, leading to habituation of anxiety and providing further evidence to contradict maladaptive appearance-related beliefs. ${ }^{39}$

The small number of controlled studies investigating CBT and selective serotonin reuptake inhibitors as treatments for BDD limited the extent to which the data can be generalized and indicates a need for the earlier controlled studies to be replicated with different samples. For instance, although a meta-analysis reported an advantage of CBT over pharmacotherapy, medication status was frequently not described and may have affected treatment outcomes. Clearly, the lack of blinding and lack of rigorous control groups in the CBT arms give $\mathrm{CBT}$ an advantage in a meta-analysis comparing the two types of treatment. Because there is no study comparing CBT with selective serotonin reuptake inhibitors or CBT plus selective serotonin reuptake inhibitors in BDD, we are unable to recommend combined treatment of these modalities. However, as clinicians, we acknowledge that, 
in some circumstances (eg, very severe, impulsive, and/or suicidal cases), it might be pertinent, or even unavoidable, to associate $\mathrm{CBT}$ and medication in the treatment of BDD.

Currently, CBT is considered the first choice for psychotherapeutic treatment of the majority of disorders in the obsessive-compulsive spectrum (including BDD), with variations in the degree to which each of the disorders could benefit from such treatment. ${ }^{43,44}$ Findings based on case series, open studies, and controlled studies, as well as the fact that several disorders with characteristics similar to BDD (eg, obsessive-compulsive disorder, social phobia, eating disorders, and depression) have been shown to respond favorably to CBT, support the adoption of this therapeutic method as the first choice among the psychotherapies recommended for patients with BDD..$^{43}$ However, it should be stressed that no other forms of psychological therapies have been studied in BDD except in small case series.

The cognitive-behavioral techniques used to treat BDD follow guidelines similar to the techniques used to treat obsessive-compulsive disorder (eg, ERP) but also have many particularities, as reviewed above. There are solidly validated data that confirm the efficacy of CBT for the treatment of obsessive-compulsive disorder, ${ }^{45}$ especially in certain groups of patients who manage to establish a good therapeutic alliance, reduce anxiety, and understand the performance of ERP tasks immediately after the beginning of treatment. This reinforces the importance of performing studies with the goal of identifying the impact of certain characteristics, such as the types of imaginary defects or the number and type of pretreatment comorbidities, on a patient's response to treatment for BDD.

One could argue that a total of 14 selected articles is relatively low as compared with the hundreds of articles initially found by our search strategy. However, this discrepancy might have resulted from the rather broad approach adopted and the fact that the diagnosis of BDD is relatively new compared with other well known psychiatric conditions. The fact that BDD was only added to the official diagnostic criteria (DSM-III-R) in 1987 may have hindered interest on the syndrome, thus delaying the publication of treatment studies on the subject.

Some methodological limitations in our study are inherent to a systematic qualitative review and can only be resolved by quantitative analyses capable of revealing more precisely the efficacy of each therapeutic approach reviewed here. However, we should also point out that the reduced number of quality studies focusing on BDD is a factor that significantly compromises the quality of information resulting from a meta-analysis. The small sample sizes of all the eligible studies examined in this review also seems like a limitation that should be mentioned.

New randomized head-to-head clinical trials that involve more homogenous treatment techniques (including individual components of CBT), active control groups, standardized evaluation measures, patients with different degrees of insight and comorbidities, and short-term and long-term follow-up analysis need to be developed to clarify the effects that the different methods of psychotherapeutic and pharmacological treatments have on BDD.

\section{Conclusion}

We reviewed the published evidence suggesting that CBT may be capable of improving symptoms of BDD in adult patients. However, the findings are limited by the small number of controlled studies that shared the same methodology and by the absence of control groups involving treatments based on the patient-therapist relationship. Clearly, comparison groups should control for nonspecific treatment effects, like therapist attention and time. Current studies do not allow us to ascertain the real effect of each cognitive and behavioral intervention, what specific conditions of cognitive behavioralbased therapies may optimize pharmacological treatment, and which patients are most likely to benefit from CBT.

\section{Disclosure}

The authors report no conflicts of interest in this work.

\section{References}

1. Pope HG Jr, Phillips KA, Olivardia R. The Adonis Complex: The Secret Crisis of Male Body Obsession. New York, NY: Free Press; 2000.

2. Harris DL, Carr AT. Prevalence of concern about physical appearance in the general population. Br J Plast Surg. 2001;54(3):223-226.

3. Liao Y, Knoesen NP, Deng Y, et al. Body dysmorphic disorder, social anxiety and depressive symptoms in Chinese medical students. Soc Psychiatry Psychiatr Epidemiol. 2010;45(10):963-971.

4. Castle DJ, Rossell SL. An update on body dysmorphic disorder. Curr Opin Psychiatry. 2006;19(1):74-78.

5. Castle DJ, Rossell S, Kyrios M. Body dysmorphic disorder. Psychiatr Clin North Am. 2006;29(2):521-538.

6. World Health Organization. The ICD-10 Classification of Mental and Behavioral Disorders. Geneva, Switzerland: World Health Organization; 1992.

7. Faravelli C, Salvatori S, Galassi F, Aiazzi L, Drei C, Cabras P. Epidemiology of somatoform disorders: a community survey in Florence. Soc Psychiatry Psychiatr Epidemiol. 1997;32(1):24-29.

8. Koran LM, Abujaoude E, Large MD, Serpe RT. The prevalence of body dysmorphic disorder in the United States adult population. CNS Spectr. 2008;13(4):316-322.

9. Phillips KA, Coles ME, Menard W, Yen S, Fay C, Weisberg RB. Suicidal ideation and suicide attempts in body dysmorphic disorder. J Clin Psychiatry. 2005;66(6):717-725.

10. Fontenelle LF, Telles LL, Nazar BP, et al. A sociodemographic, phenomenological, and long-term follow-up study of patients with body dysmorphic disorder in Brazil. Int J Psychiatry Med. 2006;36(2):243-259. 
11. Buhlmann U, Glaesmer H, Mewes R, et al. Updates on the prevalence of body dysmorphic disorder: a population-based survey. Psychiatry Res. 2010;178(1):171-175.

12. Phillips KA, Menard W, Pagano ME, Fay C, Stout RL. Delusional versus nondelusional body dysmorphic disorder: clinical features and course of illness. J Psychiatr Res. 2006;40(2):95-104.

13. Labuschagne I, Castle DJ, Dunai J, Kyrios M, Rossell SL. An examination of delusional thinking and cognitive styles in body dysmorphic disorder. Aust N Z J Psychiatry. 2010;44(8):706-712.

14. Phillips KA, Menard W, Fay C, Pagano ME. Psychosocial functioning and quality of life in body dysmorphic disorder. Compr Psychiatry. 2005;46(4):254-260.

15. Didie ER, Menard W, Stern AP, Phillips KA. Occupational functioning and impairment in adults with body dysmorphic disorder. Compr Psychiatry. 2008;49(6):561-569.

16. Wylie KR, Eardley I. Penile size and the 'small penis syndrome'. BJU Int. 2007;99(6):1449-1455.

17. Grant JE, Menard W, Pagano ME, Fay C, Phillips KA. Substance use disorders in individuals with body dysmorphic disorder. $J$ Clin Psychiatry. 2005;66(3):309-316.

18. Pinto A, Phillips KA. Social anxiety in body dysmorphic disorder. Body Image. 2005;2(4):401-405.

19. Coles ME, Phillips KA, Menard W, et al. Body dysmorphic disorder and social phobia: cross-sectional and prospective data. Depress Anxiety. 2006;23(1):26-33.

20. Castle DJ, Phillips KA, Dufresne RG Jr. Body dysmorphic disorder and cosmetic dermatology: more than skin deep. J Cosmet Dermatol. 2004;3(2):99-103.

21. Sarwer DB, Crerand CE. Body dysmorphic disorder and appearance enhancing medical treatments. Body Image. 2008;5(1):50-58.

22. Phillips KA, Hollander E. Treating body dysmorphic disorder with medication: Evidence, misconceptions, and a suggested approach. Body Image. 2008;5(1):13-27.

23. Phillips KA, Rogers J. Cognitive-behavioral therapy for youth with body dysmorphic disorder: current status and future directions. Child Adolesc Psychiatr Clin N Am. 2011;20(2):287-304.

24. Shorter E. A Historical Dictionary of Psychiatry. Oxford, UK: Oxford University Press; 2005.

25. Rachman S. Psychological treatment of anxiety: The evolution of behavior therapy and cognitive behavior therapy. Annu Rev Clin Psychol. 2009;5:97-119.

26. Rush A, Beck A, Kovacs M, Hollon S. Comparative efficacy of cognitive therapy and pharmacotherapy in the treatment of depressed outpatients. Cognit Ther Res. 1977;1:17-37.

27. Greenberg JL, Wilhelm S. Cognitive-behavioral therapy for body dysmorphic disorder: a review and future directions. Int J Cognit Ther. 2011;4(4):349-362.

28. Veale D. Advances in a cognitive behavioural model of body dysmorphic disorder. Body Image. 2004;1(1):113-125.

29. Wilhelm S, Phillips KA, Fama JM, Greenberg JL, Steketee G. Modular cognitive-behavioral therapy for body dysmorphic disorder. Behav Ther. 2011;42(4):624-633.
30. Allen LA, Woolfolk RL. Cognitive behavioral therapy for somatoform disorders. Psychiatr Clin North Am. 2010;33(3):579-593.

31. Williams J, Hadjistavropoulos T, Sharpe D. A meta-analysis of psychological and pharmacological treatments for body dysmorphic disorder. Behav Res Ther. 2006;44(1):99-111.

32. Ipser JC, Sander C, Stein DJ. Pharmacotherapy and psychotherapy for body dysmorphic disorder. Cochrane Database Syst Rev. 2009; 1:CD005332.

33. Neziroglu F, McKay D, Todaro J, Yariura-Tobias JA. Exposure, response prevention, and cognitive therapy of body dysmorphic disorder. Behav Ther. 1996;27:67-77.

34. Veale D, Gournay K, Dryden W, et al. Body dysmorphic disorder: a cognitive behavioural model and pilot randomised controlled trial. Behav Res Ther. 1996;34(9):717-729.

35. Wilhelm S, Otto MW, Lohr B, Deckersbach T. Cognitive behavior group therapy for body dysmorphic disorder: a case series. Behav Res Ther. 1999;37(1):71-75.

36. Rosen JC, Reiter J, Orosan P. Cognitive-behavioral body image therapy for body dysmorphic disorder. J Consult Clin Psychol. 1995; 63(2):263-269.

37. McKay D, Todaro J, Neziroglu F, Campisi T, Moritz EK, YaryuraTobias JA. Body dysmorphic disorder: A preliminary evaluation of treatment and maintenance using exposure with response prevention. Behav Res Ther. 1997;35(1):67-70.

38. McKay D. Two-year follow-up of behavioral treatment and maintenance for body dysmorphic disorder. Behav Modif. 1999;23(4):620-629.

39. Geremia GM, Neziroglu F. Cognitive therapy in the treatment of body dysmorphic disorder. Clin Psychol Psychother. 2001;8(4):243-251.

40. Rabiei M, Mulkens S, Kalantari M, Molavi H, Bahrami F. Metacognitive therapy for body dysmorphic disorder patients in Iran: acceptability and proof of concept. J Behav Ther Exp Psychiatry. 2012;43(2):724-729.

41. Khemlani-Patel S, Neziroglu F, Mancusi LM. Cognitive-behavioral therapy for body dysmorphic disorder: a comparative investigation. Int J Cognit Ther. 2011;4(4):363-380.

42. Saxena S, Winograd A, Dunkin JJ, et al. A retrospective review of clinical characteristics and treatment response in body dysmorphic disorder versus obsessive-compulsive disorder. J Clin Psychiatry. 2001;62(1):67-72.

43. Allen A. Cognitive-behavioral treatment of body dysmorphic disorder. Prim Psychiatry. 2006;13(7):70-76.

44. Ravindran AV, da Silva TL, Ravindran LN, Richter MA, Rector NA. Obsessive-compulsive spectrum disorders: A review of the evidencebased treatments. Can J Psychiatry. 2009;54(5):331-343.

45. Prazeres AM, Souza WF, Fontenelle LF. Cognitive-behavior therapy for obsessive-compulsive disorder: a systematic review of the last decade. Rev Bras Psiquiatr. 2007;29(3):262-270.

46. Neziroglu F, Yaryura-Tobias JA. Exposure, response prevention, and cognitive therapy in the treatment of body dysmorphic disorder. Behav Modif. 1993;23(4):620-629.
Neuropsychiatric Disease and Treatment

\section{Publish your work in this journal}

Neuropsychiatric Disease and Treatment is an international, peerreviewed journal of clinical therapeutics and pharmacology focusing on concise rapid reporting of clinical or pre-clinical studies on a range of neuropsychiatric and neurological disorders. This journal is indexed on PubMed Central, the 'PsycINFO' database and CAS.

\section{Dovepress}

The manuscript management system is completely online and includes a very quick and fair peer-review system, which is all easy to use. Visit $\mathrm{http}: / / \mathrm{www}$.dovepress.com/testimonials.php to read real quotes from published authors. 